\title{
ANÁLISE PREMILINAR DO DESEMPENHO AERODINÂMICO DE ROTORES EÓLICOS UTILIZANDO DUPLA-PÁ
}

Rodolfo Lopes ${ }^{1}$, Alex Santos ${ }^{2}$, Luzia Tofaneli ${ }^{3}$ e Turan Dias ${ }^{4}$

${ }^{1}$ Centro Universitário SENAI CIMATEC, Salvador/Bahia; rodolforl1@gmail.com

${ }^{2}$ Centro Universitário SENAI CIMATEC, Salvador/Bahia; alex.santos@fieb.org.br

3 Centro Universitário SENAI CIMATEC, Salvador/Bahia; luzia.tofaneli@fieb.org.br

${ }^{4}$ Centro Universitário SENAI CIMATEC, Salvador/Bahia; turan.oliveira@fieb.org.br

Resumo: Com o crescimento na demanda por energia elétrica, governos tem incentivado o desenvolvimento tecnológico para ampliar a geração através de fontes renováveis, contribuindo na redução da taxa de crescimento do efeito estufa no planeta. Dessa forma, este trabalho tem como objetivo apresentar os resultados preliminares sobre aprimoramentos propostos em Turbinas Eólicas de Eixo Horizontal (TEEH) quando utilizando o conceito de dupla-pá ao invés do modelo de pá convencional através de simulações por Fluidodinâmica Computacional (CFD), avaliando o comportamento dos rotores sobre condições específicas de velocidade do vento, distribuição de torque ao longo das superfícies aerodinâmicas, além do comportamento do fluxo ao passar pelo rotor.

Palavras-Chaves: turbina eólica; dupla-pá; CFD; energia renovável.

\section{PRELIMINAR ANALYSIS OF AERODYNAMIC PERFORMANCE OF WIND ROTORS USING DOUBLE-BLADE}

Abstract: With the electricity forecast growth demand, governments have been encouraging technological development to expand generation through renewable sources, contributing to the reduction of the greenhouse growth rate on the planet. Thus, this paper aims to present the preliminary results of improvements on Horizontal Axis Wind Turbine (HAWT) rotors when using double-blade concept versus conventional blade model by Computational Fluid Dynamics (CFD) analysis, evaluating its behavior under specific conditions of wind speed, torque distribution along their aerodynamic surfaces, and flow behavior when passing through the rotor.

Keywords: wind turbine; double-blade; CFD; renewable energy. 


\section{INTRODUÇÃO}

Conforme previsões de agências internacionais é possível identificar um crescimento no uso de fontes renováveis para geração de energia elétrica até 2040 [1]. Dessa forma, políticas governamentais [2] tem sido implementadas para incentivar o uso de tecnologias que possam promover uma geração de energia menos poluente possível, proveniente de recursos que podem ser regenerados naturalmente (ex.: sol, vento e etc). O principal motivo para a indicação de sua utilização é devido à redução, ou completa ausência de emissões de gases para o meio ambiente relacionados ao efeito estufa; oposto aos combustíveis fósseis. Dessa forma, entre várias tecnologias existentes, este trabalho foi concentrado na energia eólica por ser uma das fontes que mais cresceu no mundo [3], conforme mostra a figura 1.

Figura 1: Geração de energia elétrica mundial por fonte em \% entre os anos de 1980 a 2015.

\begin{tabular}{|c|c|c|c|c|c|c|}
\hline Fontes & 1980 & 2010 & 2013 & 2014 & 2015 & $\begin{array}{c}\Delta \% \\
(2015 / 2010) \\
\end{array}$ \\
\hline Combustíveis fósseis & $69,6 \%$ & $66,3 \%$ & $66,4 \%$ & $65,9 \%$ & $65,6 \%$ & $-1,1 \%$ \\
\hline Hidrelétrica & $21,5 \%$ & $16,6 \%$ & $16,8 \%$ & $17,0 \%$ & $16,6 \%$ & $0,0 \%$ \\
\hline Nuclear & $8,5 \%$ & $12,8 \%$ & $10,6 \%$ & $10,6 \%$ & $10,6 \%$ & $-17,2 \%$ \\
\hline Biomassa e resíduos sólidos & $0,2 \%$ & $1,8 \%$ & $2,0 \%$ & $2,2 \%$ & $2,3 \%$ & $27,8 \%$ \\
\hline Geotérmica & $0,2 \%$ & $0,3 \%$ & $0,3 \%$ & $0,3 \%$ & $0,3 \%$ & $0,0 \%$ \\
\hline Solar & $0,0 \%$ & $0,1 \%$ & $0,6 \%$ & $0,9 \%$ & $1,1 \%$ & $1000,0 \%$ \\
\hline Eólica & $0,0 \%$ & $2,1 \%$ & $3,2 \%$ & $3,1 \%$ & $3,5 \%$ & $66,7 \%$ \\
\hline
\end{tabular}

Fonte: Autor com base em [3], p.35

Os equipamentos eólicos tem boa eficiência, mas por questões financeiras os fabricantes preferem aumentar o tamanho destes a trabalharem em desempenho, obtendo assim maior disponibilidade de potência e, consequentemente maior geração de eletricidade. Seu princípio de funcionamento compreende o momento da passagem do vento através do rotor do aerogerador, que captura a potência através da energia cinética contida no vento, ou seja, a extração ocorre com a diferença de pressão antes e depois do rotor [4], a exemplo em TEEH onde as forças aerodinâmicas impelem movimento ao rotor que está acoplado a um eixo ligado a um gerador de potência. No entanto, estes equipamentos não operam constantemente na máxima condição de extração devido a variação de intensidade ou nenhum deslocamento de massas de ar [5].

Pensando nisso, este trabalho apresenta os resultados preliminares em relação ao comportamento de um rotor proposto com diâmetro de $6,3 \mathrm{~m}$ utilizando duplas-pás desde sua raiz até a ponta e com a distribuição dos perfis aerodinâmicos conforme feito por [6], além de observar o fluxo ao atravessar o rotor. 
A duplicação das pás foi idealizada com base em aeronaves biplanas devido o potencial de aumentar a área de sustentação [7,8], o que pode ajudar no desempenho de extração de energia cinética pelo rotor, entretanto, com o aumento da superfície aumenta-se sua inércia que pode depender cada vez mais de maiores velocidades de vento. Para potencializar essa condição, em [9] uma turbina foi instalada entre grandes construções, fazendo valer o efeito Venturi. Já a pá convencional foi obtida a partir de [10].

\section{METODOLOGIA}

Para as simulações foi utilizado o software ANSYS CFX 18.2, além de revisão da literatura para fundamentar a execução do trabalho, obtendo soluções que representem da melhor forma o comportamento físico em turbinas eólicas. A partir desta etapa foram desenvolvidos os modelos geométricos e gerada a malha computacional para obtenção da solução numérica das equações ao escoamento estudado. A figura 2 mostra as geometrias estudas, o domínio e a malha desenvolvida.

Figura 2: Geometrias, domínio e malha computacional estudadas

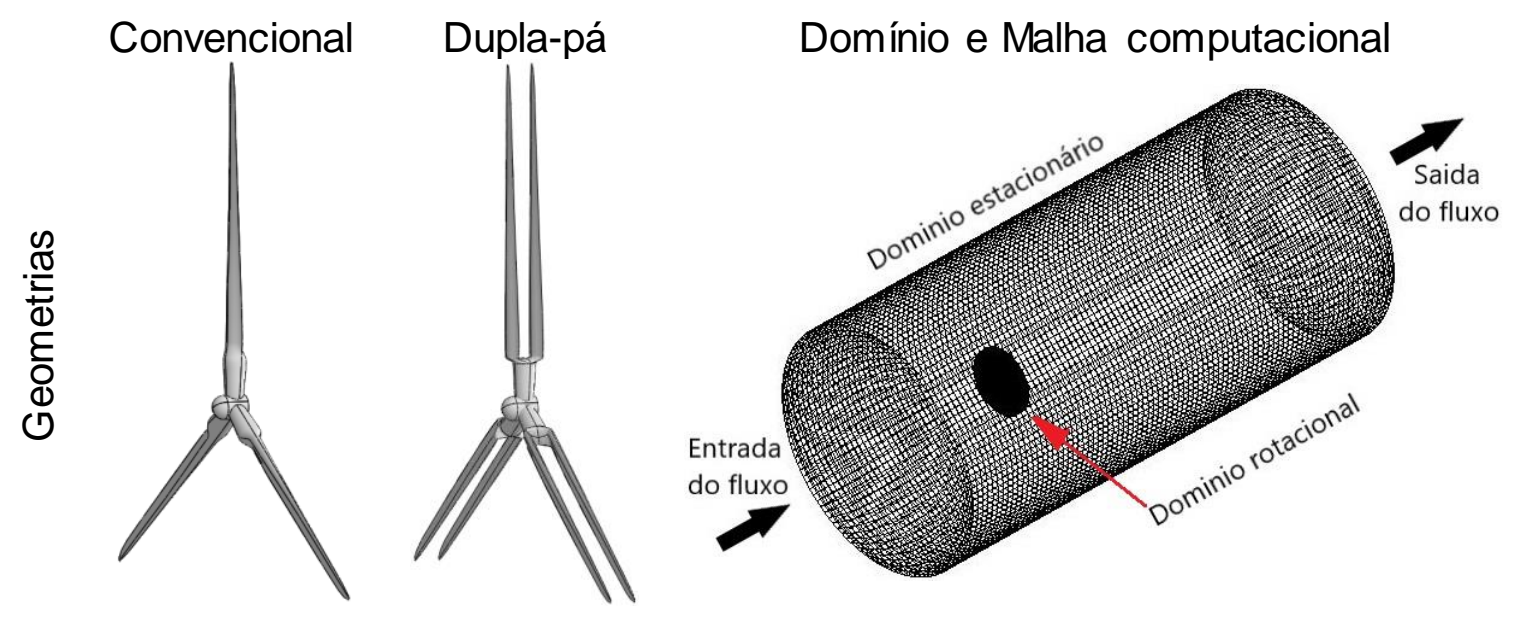

Fonte: Autor

As análises foram executadas considerando os rotores completos, utilizando o mesmo hub e perfis da raiz até $r / R=15 \%$ das pás para evitar problemas de variação naquela região. Além disso, apenas uma de suas pás para análise segmentada conforme visto em [11] que dividiu o rotor em anéis, observando suas diferenças entre os perfis propostos naquele estudo. Neste trabalho o rotor foi dividido em segmentos tal como mostra a figura 3, além dos parâmetros de projeto para os perfis aerodinâmicos (tipo, espessuras, posição e ângulo de torção) conforme [6,10] e do ângulo de instalação das pás nos rotores em zero grau em relação ao plano de rotação que representa a máxima condição de geração de potência naquele rotor. 
Figura 3: Planos e seções de apresentação dos resultados

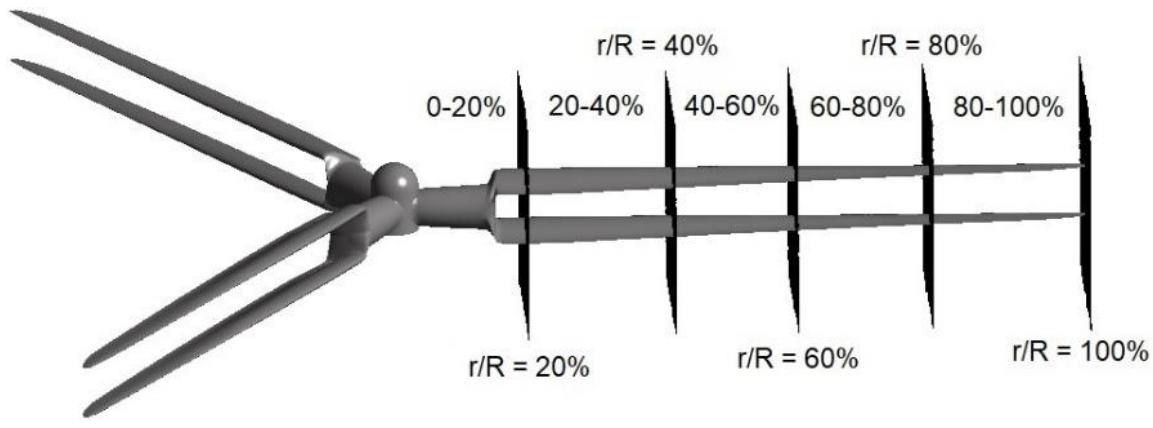

Fonte: Autor

\subsection{Modelagem matemática}

Para atingir a solução que descreve o escoamento estudado, foram utilizadas as equações de continuidade (1) e de Navier-Stokes (2) a (4), além de um modelo de turbulência. Neste trabalho foi utilizado o modelo SST devido ser adequado a escoamentos externos com proximidade a superfície analisada [12].

$$
\begin{gathered}
\frac{\partial \rho}{\partial t}+\operatorname{div}(\rho \vec{u})=0 \\
\frac{\partial(\rho u)}{\partial t}+\nabla \cdot(\rho u \vec{U})=-\frac{\partial p}{\partial x}+\nabla \cdot(\mu \nabla u)+S_{M x} \\
\frac{\partial(\rho v)}{\partial t}+\nabla \cdot(\rho v \vec{U})=-\frac{\partial p}{\partial y}+\nabla \cdot(\mu \nabla v)+S_{M y} \\
\frac{\partial(\rho w)}{\partial t}+\nabla \cdot(\rho w \vec{U})=-\frac{\partial p}{\partial z}+\nabla \cdot(\mu \nabla w)+S_{M w}
\end{gathered}
$$

onde, $p$ é a pressão, $\vec{U}$ é o vetor tridimensional de velocidades, formado pelas componentes $(u, v, w), \rho$ a massa específica, $\mu$ a viscosidade dinâmica do fluido e $t$ o tempo. E $S_{M}$, são termos das fontes geradoras de momento.

As condições de contorno adotadas no modelo foram baseadas em [10] com os domínios contendo ar a $25^{\circ} \mathrm{C}$ (condição isotérmica), pressão de $1 \mathrm{~atm}$ e velocidade do vento variando de 5 a $7 \mathrm{~m} / \mathrm{s}$. A interface entre os domínios foi configurada como rotacional, com a rotação do rotor fixada em 121rpm. Além do escoamento sendo considerado como regime permanente. 


\section{RESULTADOS E DISCUSSÃO}

A partir da metodologia apresentada, os resultados foram obtidos para: distribuição do torque ao longo da pá, comportamento do fluxo no domínio e em uma das pás de cada rotor. Para a distribuição do torque, foram encontrados valores próximos no segmento $0-20 \%$ entre os rotores já que tem geometria similar. Nos seguimentos posteriores $(20-40 \%$ e $40-60 \%)$ foi observada a queda nos valores de torque no rotor com duplas-pás devido a menor espessura nos perfis utilizados em relação ao rotor convencional. E a partir de $60 \%$ à ponta, onde os segmentos são igualmente duplicados é observado que os valores são maiores na dupla, como mostra a figura 4 devido a ter maior área superficial.

Figura 4: Distribuição de torque na pá nas velocidades 5,0 e 7,0m/s

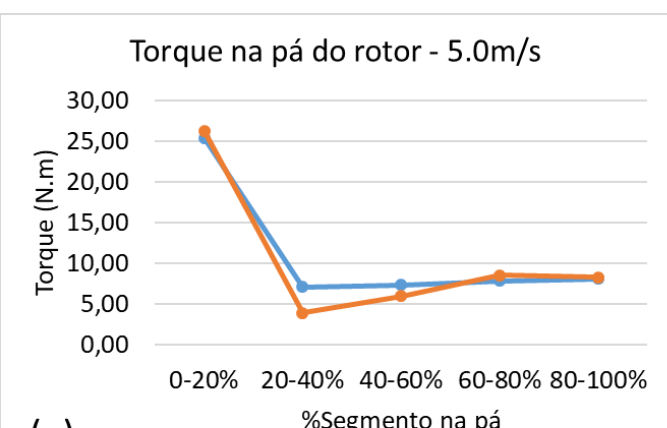

(a)

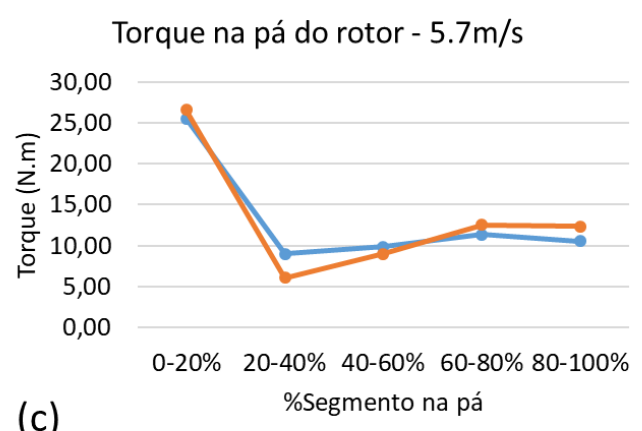

(c)

Torque na pá do rotor $-7.0 \mathrm{~m} / \mathrm{s}$

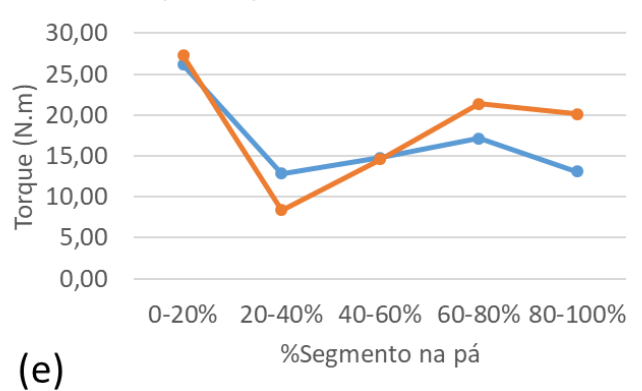

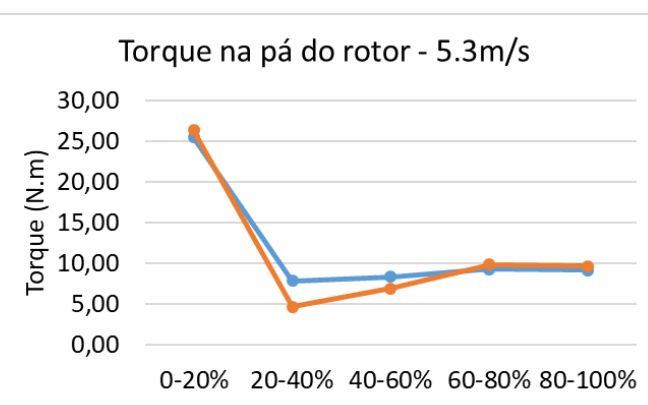

(b)

\%Segmento na pá

Torque na pá do rotor $-6.5 \mathrm{~m} / \mathrm{s}$

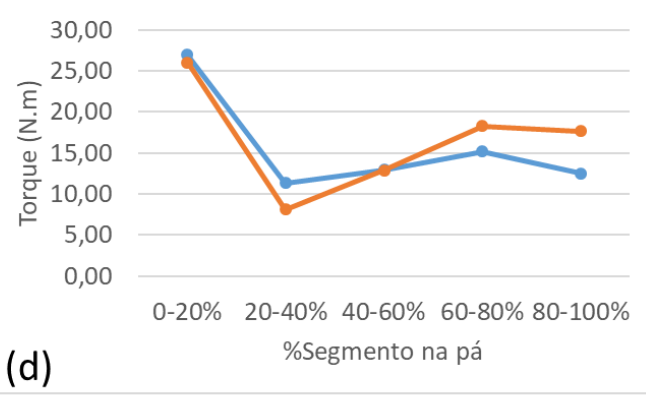

(d)

Fonte: Autor 


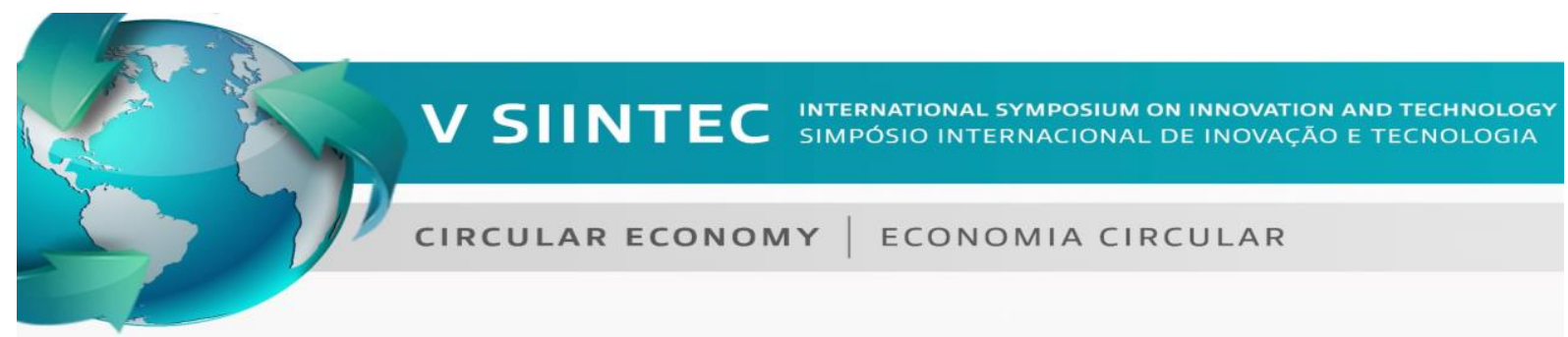

Para as linhas de fluxo que passam através dos rotores, foi observado que a partir de ventos com velocidades acima de $5,7 \mathrm{~m} / \mathrm{s}$, ocorrem maiores valores de torque no rotor com dupla-pá, além de maior desaceleração do fluxo à montante do que na pá convencional devido a presença do rotor em si, e a extração da potência disponível é ampliada com o aumento da área superficial, como mostra a figura 5 . O efeito visual identificado na separação das linhas do fluxo à jusante também mostra a condição de desaceleração do fluxo. Assim, quanto mais distantes as linhas de fluxo, menor será sua velocidade após o rotor.

Figura 5: Linhas de fluxo através do rotor
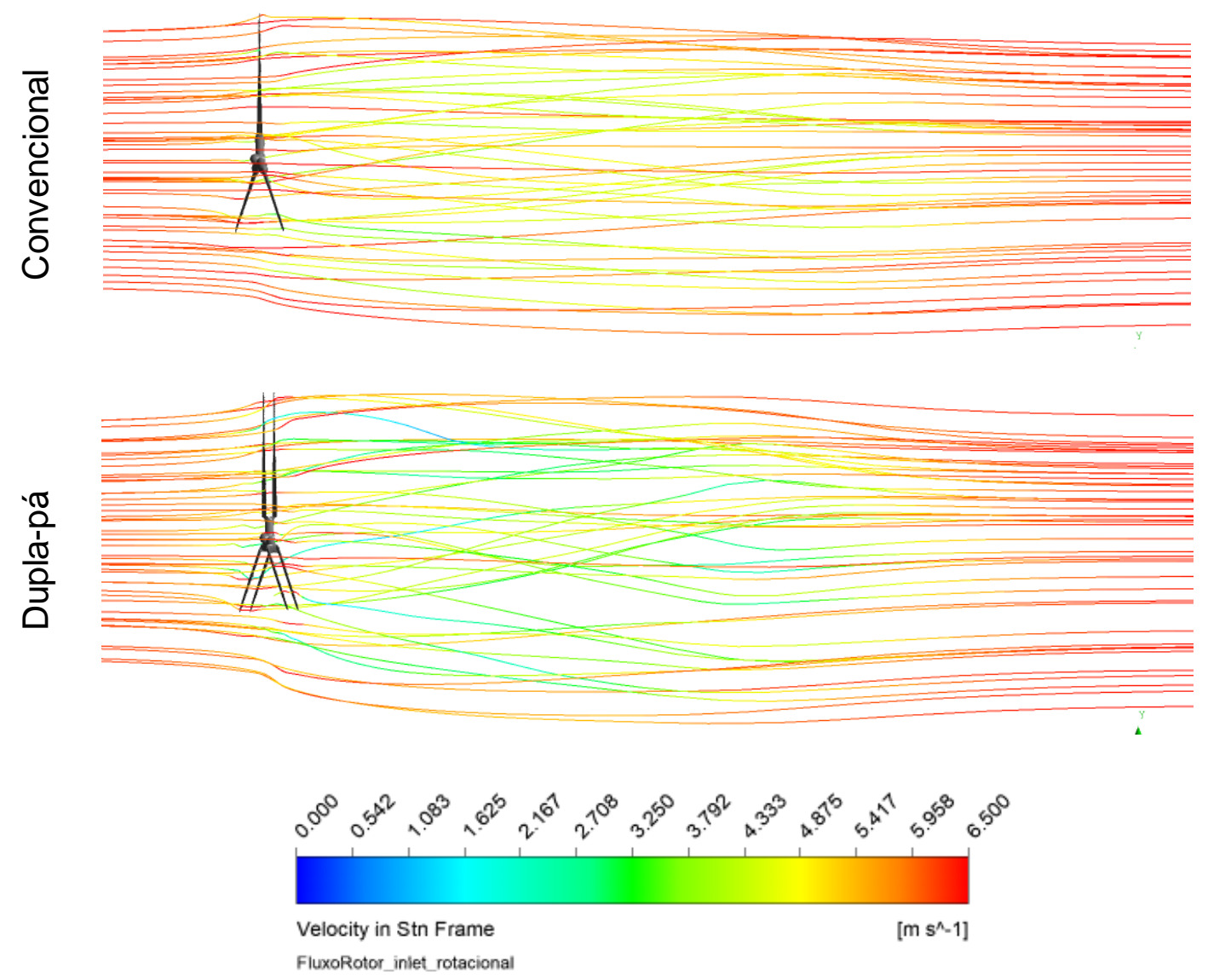

Fonte: Autor

Outra forma de visualizar as linhas de fluxo é demonstrá-las através de uma das pás do rotor, explicando assim o porquê das maiores extrações de potência no rotor com duplas-pás, pois quando o fluxo atravessa a superfície aerodinâmica do rotor com pá convencional, este passa apenas por uma superfície, e retorna a condição do fluxo livre de forma mais rápida, porém na dupla-pá, passa da primeira 
superfície à montante, e após, da segunda à jusante, ou seja, mais uma parcela de energia embora menor também será extraída, provocando maior redução na velocidade do vento, conforme mostra a figura 6 .

Figura 6: Linhas de fluxo através da pá do rotor

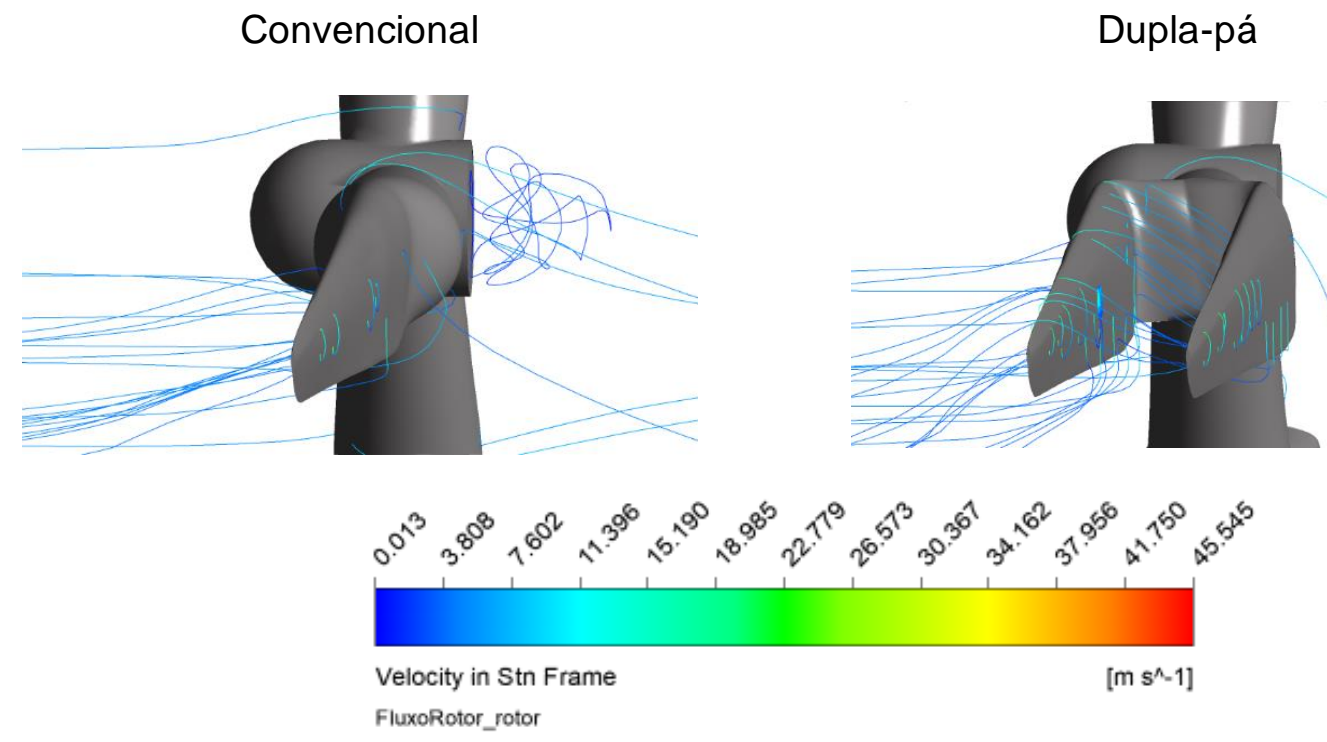

Fonte: Autor

\section{CONCLUSÃO}

Esta análise preliminar conseguiu desenvolver as primeiras simulações utilizando CFD nos modelos geométricos, iniciando 0 entendimento do comportamento do fluxo em torno dos rotores. Para o rotor com duplas-pás, demonstrou resultados $6 \%$ melhores em média na distribuição de torque nas superfícies aerodinâmicas. Entretanto é possível observar que se faz necessário melhorar o refino da malha em algumas regiões, tal como bordo de fuga e ponta de pá em ambas as superfícies, principalmente na duplicação por ter perfis mais finos e com maior cambagem. Também se faz necessário complementar as avaliações dos perfis nas regiões duplicadas para velocidade e pressão nos planos definidos. Além disso, foi possível identificar que em um rotor utilizando duplas-pás, seu local de instalação requer mais detalhamento e deverá ter um potencial eólico maior para obter melhores resultados na geração de potência em relação ao rotor com pá convencional. 


\section{REFERÊNCIAS}

1BP Energy Economics, "2018 BP Energy Outlook 2018 BP Energy Outlook," p. 125, 2018.

2United Nations, The Sustainable Development Goals Report 2016. UN, 2016.

3MINISTÉRIO DE MINAS E ENERGIA. EMPRESA de PESQUISA ENERGÉTICA (EPE), "Anuário Estatístico de Energia Elétrica 2018 no ano base de 2017," p. 249, Brasilia-DF, 2018.

${ }^{4}$ T. Burton, N. Jenkins, D. Sharpe, and E. Bossanyi, Wind Energy Handbook. $2^{\mathrm{a}}$ ed. Chichester, West Sussex: Wiley, 2011.

${ }^{5} \mathrm{~K}$. Grogg and C. College, "Harvesting the Wind: The Physics of Wind Turbines," Wind Energy, pp. 1-44, 2005.

${ }^{6} \mathrm{P}$. Chiu, "Aerodynamics and optimal design of biplane wind turbine blades," Tese de doutorado - University of California, Los Angeles, 2017.

${ }^{7}$ L. E. M. J. RODRIGUES, Fundamentos da engenharia aeronáutica aplicações ao projeto sae-aerodesign, 1a ed. Salto/SP, 2014.

${ }^{8}$ R. W. Derksen and A. G. Kraj, "Aerodynamic optimization of a biplane configuration using differential evolution,” Comput. Aided Optim. Des. Eng. X, vol. I, pp. 209-218, 2007.

${ }^{9}$ Y. G. Heo, N. J. Choi, K. H. Choi, H. S. Ji, and K. C. Kim, "CFD study on aerodynamic power output of a $110 \mathrm{~kW}$ building augmented wind turbine," Energy Build., vol. 129, pp. 162-173, 2016.

10J. Jonkman, S. Butterfield, W. Musial, and G. Scott, "Definition of a 5-MW Reference Wind Turbine for Offshore System Development," no. February, 2009.

${ }^{11} \mathrm{~J}$. Laursen, P. Enevoldsen, and S. Hjort, "3D CFD quantification of the performance of a multi-megawatt wind turbine," J. Phys. Conf. Ser., vol. 75, no. 1, 2007.

${ }^{12} \mathrm{C}$. J. Bai and W. C. Wang, "Review of computational and experimental approaches to analysis of aerodynamic performance in horizontal-axis wind turbines (HAWTs),"

Renew. Sustain. Energy Rev., vol. 63, pp. 506-519, 2016. 